\title{
Investigation of interfacial thermal transport across graphene and an organic semiconductor using molecular dynamics simulations
}

\author{
Xinyu Wang, ${ }^{a}$ Jingchao Zhang, ${ }^{b}$ Yue Chen ${ }^{* a}$ and Paddy K. L. Chan ${ }^{* a}$ \\ ${ }^{a}$ Department of Mechanical Engineering, The University of Hong Kong, Hong Kong \\ ${ }^{\mathrm{b}}$ Holland Computing Center, University of Nebraska-Lincoln, Lincoln, NE 68588, USA
}

\begin{abstract}
The interfacial thermal transport across graphene and organic semiconductor, dinaphtho[2,3-b:2',3'-f]thieno[3,2-b]thiophene (DNTT) is investigated by molecular dynamics simulation. The average thermal boundary resistance (TBR) of graphene and DNTT is $4.88 \pm 0.12 \times 10^{-8} \mathrm{~m}^{2}-\mathrm{K} / \mathrm{W}$ at $300 \mathrm{~K}$. We find that TBR of graphene-DNTT heterostructure possesses the as high as $83.4 \%$ reduction after the hydrogenation of graphene. Moreover, as graphene vacancy increases from $0 \%$ to $6 \%$, the TBR drops up to $39.6 \%$. The reduction of TBR mainly attributes to the coupling enhancement of the graphene and DNTT phonons as evaluated from the phonon density of states. On the other hand, TBR keeps a constant value while the vacancy in the DNTT layer is increasing. The TBR would decrease when the temperature and coupling strength increase. These findings provide a useful guideline to the thermal management of the graphene-based organic electronic devices, especially for the large area transistor arrays or sensors.
\end{abstract}

\section{Introduction}

The unique electrical and thermal properties of graphene open a new research area in flexible electronics for both active layers and electrodes. ${ }^{1-6}$ For example, the graphene-based transistors not only operate at gigahertz frequencies as high as 100 $\mathrm{GHz},{ }^{7,8}$ but also possess a high carrier mobility ${ }^{2}$ around $10000 \mathrm{~cm}^{2} / \mathrm{V}$-s, which shows

\footnotetext{
"Corresponding authors. E-mail: pklc@hku.hk (Paddy K. L. Chan) and yuechen@hku.hk (Yue Chen).

$\dagger$ Electronic Supplementary Information (ESI) available. See DOI: 10.1039/x0xx00000x
} 
tremendous potential in nanoscale electronic devices. The superior in-plane thermal conductivity of graphene also allows effective heat dissipation for the electronic devices. Theoretical and experimental investigations have demonstrated that in-plane thermal conductivity of the graphene can reach $2000-6000 \mathrm{~W} / \mathrm{m}-\mathrm{K}$ measured or calculated by using the confocal micro-Raman spectroscopy, ${ }^{6}$ suspended micro-bridge method, ${ }^{9}$ molecular dynamics simulation ${ }^{9}$ and first-principles calculations. ${ }^{10}$ These physical properties of graphene have been further optimized and investigated under different structures including nanoribbons, ${ }^{11}$ stacked bilayers, ${ }^{12}$ or multilayer, ${ }^{2}$ or chemical treatment. $^{13,14}$

Other than the pure graphene-based investigation, its interaction with other semiconductors primarily organic semiconductors has also become a popular research topic in the last decade. Organic active layers with graphene as the conductive electrodes have been further broadening the horizons of this new class flexible electronics. ${ }^{15-17}$ One important aspect of such graphene-organic device is the energy band alignment. Park et al. reported that the work function of graphene could be optimized by functionalizing the substrate with self-assembled monolayers (SAMs) and reduce the charge injection barrier in organic field effect transistors (OFETs). ${ }^{15}$ On the other hand, the carrier mobility in the polymer OFETs could be increased 20 times by embedding graphene flakes into the polymer active layer. ${ }^{18}$ The performance of these contacting electrodes or active layers is strongly depending on the interface quality between the organic semiconductor and the graphene. While the current density and functionality of these graphene-organic hybrid devices are increasing, the generation of wasted heat in these devices would become critical to the overall performance. As we know the molecular alignment and surface morphology of the organic semiconductors are sensitive to the thermal environment of devices, ${ }^{19-21}$ inferior heat transportation would directly affect the device lifetime. Given that graphene has the excellent thermal conductivity, the mechanism of heat propagating through this graphene-organic interface would be the critical parameter in governing the heat dissipation in these devices. Furthermore, different from the graphene-inorganic interfaces which have been simulated ${ }^{22-25}$ or measured, ${ }^{26}$ the investigation about an interfacial thermal 
transport of graphene-organic semiconductors is still very limited.

Molecular dynamics (MD) simulation has widely been applied to investigate the thermal properties of graphene-based structures, which can not only overcome the measurement difficulties of experiment but also provide the atomic-level insight into thermal transport. ${ }^{3,27}$ Other than the intrinsic thermal properties of graphene with defects, ${ }^{28-30}$ doping $^{31,32}$ or chemical functionalization ${ }^{33-36}$ predicted by MD, the in-plane and out-of-plane interfacial thermal transports between graphene and other materials have also been calculated by MD simulation, ${ }^{23,25,26,37-42}$ which provide the useful thermal knowledge for the application of graphene. In this work, we focus on investigating the interfacial thermal transport of graphene and an air-stable small molecule organic semiconductor, dinaphtho[2,3-b:2',3'-f]thieno[3,2-b]thiophene (DNTT), using classical MD simulation. OFETs with DNTT active layer have been showing the promising carrier mobility up to $9.9 \mathrm{~cm}^{2} /(\mathrm{V}-\mathrm{s})$ in single crystal structure ${ }^{43}$ and thermally stable up to $100{ }^{\circ} \mathrm{C}$. By using 3- $\omega$ method, we measured the thermal conductivity $(k)$ of DNTT $(k=0.45 \pm 0.06 \mathrm{~W} / \mathrm{m}-\mathrm{K})$ and suggested an approach to modulate the $k$ values by embedding silver nanoparticles. ${ }^{44}$ Very recently, we utilized molecular dynamics to simulate the thermal conductivity of DNTT and obtained the bulk thermal conductivities of DNTT along the $a^{*}, b^{*}$ and $c^{*}$ directions as $0.73,0.33$ and $0.95 \mathrm{~W} /(\mathrm{m}-\mathrm{K}) .{ }^{45}$ Meanwhile, the thermal boundary resistances across different orientation interfaces were calculated as $7.00 \pm 0.26,6.15 \pm 0.13$ and $3.20 \pm 0.09 \times 10^{-9} \mathrm{~m}^{2}$ $\mathrm{K} / \mathrm{W}$ for the $a^{*}-b^{*}, a^{*}-c^{*}$ and $b^{*}-c^{*}$ interfaces, respectively. Here we apply a transient heating method in the MD simulation to mimic the experimental pump-probe technique and evaluate the thermal boundary resistance (TBR) between graphene and DNTT. We firstly study the effects of graphene dimension and DNTT thickness on the TBR values. Secondly, we investigate the effects of graphene hydrogenation and vacancy concentration. We further extend the work by simulating the TBR at different temperature and interfacial coupling strength between graphene and DNTT. The phonon density of states (DOS) of graphene and DNTT are also calculated respectively to analyze the TBR results. 


\section{Theory and modeling}

The MD simulations are performed with the Large-scale Atomic/Molecular Massively Parallel Simulator (LAMMPS) package. ${ }^{46}$ The second-generation reactive empirical bond order (REBO) potential has been reported to model C-C interaction ${ }^{47}$ in graphene and accurately predict the thermal properties of graphene. $5,22,23,48,49$ Meanwhile, various work has demonstrated that the general AMBER force field $(\mathrm{GAFF})^{50}$ are able to account for the intermolecular and intramolecular interactions of small molecule organic semiconductors, such as pentacene, ${ }^{51,}{ }^{52}$ DNTT, $^{45}$ and 2,7Dioctyl[1]benzothieno[3,2-b][1]benzothiophene (C8-BTBT). ${ }^{53}$ Therefore, we employ REBO potential to describe the C-C interaction in graphene and adopt GAFF to describe the bond, angle, dihedral, van der Waals and electrostatic interactions in the DNTT molecules. In addition, Lennard-Jones (L-J) potential is employed to describe the van der Waals interaction between graphene and DNTT as below:

$$
E_{L J}=4 \chi \varepsilon\left[\left(\frac{\sigma}{r}\right)^{12}-\left(\frac{\sigma}{r}\right)^{6}\right]
$$

where, $r$ is the distance between atoms; $\varepsilon$ is the depth of potential well; $\sigma$ is the zero energy separation distance; and $\chi$ is the coupling strength factor to adjust the interaction strength between graphene and DNTT. The L-J potential parameters are calculated from the universal force field (UFF). ${ }^{54}$ The details of the GAFF and UFF parameters used in the simulation are summarized in the ESI $\dagger$. The development of the DNTT molecules in the system is based on the DNTT structure reported by Yamamoto and Takimiya. ${ }^{55}$ In the simulation, graphene is perpendicularly placed on the $c$ direction of DNTT crystal. The zigzag direction of graphene and $a$ direction of DNTT lattice are along $x$ direction of the simulation box while the armchair direction of graphene and $b$ direction of DNTT lattice are along $y$ direction of the simulation box.

To calculate the thermal boundary resistance between graphene and DNTT, a transient heating method ${ }^{22,25,49,56,57}$ which analogizes the experimental pump-probe method, is applied in the simulation. As shown in Fig. 1(a), an ultrafast thermal impulse is imposed on the graphene, and thus the energy and temperature of graphene will be increased rapidly. During the subsequent thermal relaxation process, the thermal energy 
will propagate from graphene to DNTT. Therefore, energy and temperature of graphene will drop gradually whereas the energy and temperature of DNTT go up as shown in Fig. 1(b). The interfacial thermal transport during thermal relaxation process will obey the following equation:

$$
\frac{\partial E_{G r a}}{\partial t}=A \cdot \frac{T_{G r a}-T_{D N T T}}{R}
$$

where, $E_{G r a}$ is the energy of graphene; $T_{G r a}, T_{D N T T}$ are the temperatures of graphene and DNTT monolayer next to the interface; $t$ is the time; $A$ is the graphene area; and $R$ is the TBR. We can integrate Eq. (2) to obtain:

$$
E_{G r a, t}-E_{G r a, 0}=\frac{A}{R} \cdot \int_{0}^{t}\left(T_{G r a}-T_{D N T T}\right) d t
$$

The inset of Fig. 1(b) shows the relation curve of $E_{\text {Gra }}$ and $\int_{0}^{t}\left(T_{G r a}-T_{D N T T}\right) d t$, which demonstrates that the relation is close to linear. By linear fitting and knowing the graphene area, the TBR values can be evaluated from the slope of Eq. (3).

In our MD simulations, the periodic boundary conditions along $x$ and $y$ directions and free boundary condition along $z$ direction are used and the time step is chosen to be 0.5 fs. To develop and apply periodic boundary conditions along $x$ and $y$ directions, the lattice constant of graphene is modified, which induces a lattice modification of $0.602 \%$ along $x$ direction and $-0.102 \%$ along $y$ direction for the structure of graphene. This modification has the negligible effect on the TBR values and will be discussed later. The graphene atoms are initially positioned at $3.4 \AA$ on top of DNTT molecules. In the beginning, the system is equilibrated in a canonical (NVT) ensemble for $0.5 \mathrm{~ns}$ at the specified temperature. Afterward, a microcanonical (NVE) ensemble is performed on the system for another $0.5 \mathrm{~ns}$. When the system reaches the NVE equilibrium, an ultrafast thermal impulse $\left(8 \times 10^{12} \mathrm{~W} / \mathrm{m}^{2}\right)$ is exerted on the graphene. By monitoring the energy and temperature during the following $0.5 \mathrm{~ns}$, the TBR can be extracted based on the fitting method discussed above. For each TBR value, more than three independent simulations with different initial conditions are performed. The TBR values are calculated by averaging each simulation with the error bar as the standard deviation. To verify the effect of the DNTT orientation, we build the structures of graphene placed on the $a$ and $b$ directions of DNTT crystal. No obvious TBR variation can be observed 
at different DNTT orientations and the results are shown Fig. S1 in the ESI†. In the following parts, all the simulations are based on the structure of graphene placed on the $c$ direction of DNTT.

To reveal the interfacial energy transport physics and interpret the TBR results of graphene and DNTT, the phonon DOS is calculated by taking the Fourier transform of the velocity autocorrelation function (VACF): $:^{3,49,56}$

$$
P(\omega)=\frac{1}{\sqrt{2 \pi}} \int_{-\infty}^{\infty} e^{i \omega t} \frac{\langle\boldsymbol{v}(t) \cdot v(0)\rangle}{\langle\boldsymbol{v}(0) \cdot v(0)\rangle} d t
$$

where $P(\omega)$ denotes the phonon DOS at frequency $\omega$, and $\boldsymbol{v}(t)$ and $\boldsymbol{v}(0)$ are atom velocity vectors at $t$ time and zero time, respectively. According to the value of phonon DOS, we can analyze the energy transport mechanism of graphene and DNTT. Meanwhile, the overlapping of phonon DOS of graphene and DNTT is an indicator of the phonon transmission capacity across the interface between graphene and DNTT. To compare the phonon DOS in the same baseline, we firstly normalize the total phonon DOS areas of graphene and DNTT to 1, respectively. To quantify phonon transmission capacity, we adopt a phonon overlapping factor (arbitrary unit), which is defined as $\delta=$ $\int H(\omega) d \omega,{ }^{58}$ where $H(\omega)$ represents the intersection height of normalized phonon DOS at frequency $\omega$, to analyze the TBR results. The total intersection area of phonon DOS is proportional to the amount of energy transported across the interface.

\section{Results and discussion}

\subsection{Effects of graphene dimension and DNTT thickness}

As we know, due to the long phonon mean free path (MFP) of graphene, the inplane thermal conductivity of graphene is strongly depending on the dimension. ${ }^{59}$ To investigate effects of graphene dimension and DNTT thickness on the interfacial thermal transport between graphene and DNTT, we develop the simulation dimension with different graphene areas and DNTT thicknesses. In this part, the system initially is equilibrated at $300 \mathrm{~K}$. The interface coupling strength factor $(\chi)$ is set as unity.

Five sizes of graphene $(x \times y)$ are built as $2.475 \times 7.662,4.950 \times 7.662,9.899 \times$ $7.662,14.849 \times 7.662$, and $19.798 \times 7.662 \mathrm{~nm}^{2}$. The corresponding DNTT $x \times y(a \times b)$ dimension changes with graphene dimension and the thickness of DNTT ( $c$, along $z$ 
direction) is fixed $8.096 \mathrm{~nm}$. From the results shown in Fig. 2, it can be noticed that TBR values remain constant even the graphene area has an 8 -fold increase. We also investigate the dependence of TBR on DNTT thickness ( $z$ direction) by fixing the graphene dimension $\left(4.950 \times 7.662 \mathrm{~nm}^{2}\right)$ and enlarging the thickness of the DNTT layers $(8.096,11.335,16.193,24.289$, and $32.385 \mathrm{~nm})$. Similar to the graphene areas, TBR values keep relatively constant in various thickness of the DNTT. We can conclude that TBR of graphene-DNTT is irrelevant to the graphene dimension and DNTT thickness. By averaging the TBR values at different dimensions, the TBR of graphene-DNTT is $4.88 \pm 0.12 \times 10^{-8} \mathrm{~m}^{2}-\mathrm{K} / \mathrm{W}$, which is similar to TBR values of graphene-silicon, ${ }^{22}$ graphene-paraffin wax ${ }^{60}$ and graphene-octane. ${ }^{61,62}$

Fig. 3(a) shows the phonon DOS of graphene and DNTT. It can be observed that the major energy channel (overlapping region) of DNTT and graphene locates at the mediate frequency ranging from $20 \mathrm{THz}$ to $52 \mathrm{THz}$. Even though more long-wavelength (low-frequency) phonon can participate in energy transport by increasing graphene dimension and DNTT thickness, low-frequency phonon just makes a small contribution to the interfacial thermal transport between graphene and DNTT. Therefore, we cannot observe the significant size dependence of TBR for graphene-DNTT heterostructure.

To address the effects contributed from modifying the lattice constant of graphene in order to satisfy the periodic boundary condition in the model, we also repeat the simulation with pristine graphene lattice constant equal to $2.46 \AA$ and the results are shown in Fig. S2 in the ESI†. DNTT simulation system in free boundary conditions along $x$ and $y$ directions and five kinds of graphene size are simulated as well. By comparing these two kinds graphene structures, we find that it can be noticed that there is no significant variation for TBR values when the lattice of graphene is stretched $0.602 \%$ along $x$ direction while contracted $0.102 \%$ along $y$ direction. It can be concluded that this slight lattice modification of graphene cannot bring about the effect to the TBR. In the following sections, the lattice constant of graphene is modified and periodic boundary conditions are applied in the simulation.

\subsection{Effect of graphene hydrogenation}

One commonly adopted approach to functionalize the graphene is by adding in 
chemical groups such as, hydrogenation, ${ }^{13,14,35,39,63-65}$ fluorination, ${ }^{66}$ chemical function group treatment, ${ }^{36}$ to tune the electrical, ${ }^{13,14}$ thermal, ${ }^{35,64}$ mechanical $^{63}$ and magnetic ${ }^{65}$ properties of the graphene. To overcome the zero-bandgap nature of pristine graphene and achieve the desired electrical properties of graphene, hydrogen is widely used to functionalize graphene to control energy band gap, ${ }^{13}$ carrier mobility and on/off ratio of the transistor, ${ }^{13}$ and magnetic states, ${ }^{14,} 67$ where $s p^{2} \mathrm{C}$ bonding hybridization in graphene changes into $s p^{3}$ hybridization. Accompanied with the hydrogenationenhanced electrical properties, the generated thermal issues of devices are unneglectable to affect the performance and lifetime of these devices. As a result, it is essential to investigate the interfacial thermal transport between hydrogenated graphene (H-graphene) and organic semiconductor in graphene-based organic electronic devices.

The hydrogenation process is shown in the schematic structure of Fig. 4 where hydrogen atoms are added to carbon atoms of graphene on both sides alternately, and hydrogenated graphene sheet perpendicularly lies on DNTT $c$ direction. We define the hydrogen coverage ratio $(\alpha)$ as $\alpha=\frac{N_{H}}{N_{\text {Gra }}}$, where $N_{H}$ is the atom number of hydrogen and $N_{\text {Gra }}$ is the atom number of graphene. When $\alpha$ is equal to $100 \%$, the hydrogenated graphene is known as graphane. ${ }^{68}$ In order to minimize the effects of hydrogen distribution, the hydrogen atoms are added randomly to the graphene. The in-plane dimension of graphene is $4.950 \times 7.662 \mathrm{~nm}^{2}(x \times y)$ and the DNTT thickness is chosen as $8.096 \mathrm{~nm}$. The NVT equilibrium temperature is $300 \mathrm{~K}$ and $\chi$ is still maintained at 1 . Fig. 4 shows the TBR variation of H-graphene and DNTT as a function of hydrogen coverage ranging from $0 \%$ to $100 \%$. It can be noted that TBR will drop dramatically from $5.07 \pm 0.35 \times 10^{-8} \mathrm{~m}^{2}-\mathrm{K} / \mathrm{W}$ to $1.07 \pm 0.13 \times 10^{-8} \mathrm{~m}^{2}-\mathrm{K} / \mathrm{W}$ when the hydrogen coverage ratio increases from $0 \%$ to $20 \%$. When the hydrogen coverage ratio ranges from $30 \%$ to $80 \%$, the TBR reaches a constant value, around $8.4 \times 10^{-9} \mathrm{~m}^{2}-\mathrm{K} / \mathrm{W}$, which is only around $16.6 \%$ of TBR of pristine graphene and DNTT. With the hydrogen coverage ratio further increasing from $90 \%$ to $100 \%$, the TBR shows a slight enhancement. This observation agrees with the previous studies that the thermal conductivity of hydrogenated graphene drops obviously from $0 \%$ to $20 \%$, then levels off and finally 
increases. $^{34,35,64}$

The underlying physical mechanism about the TBR reduction after hydrogenation is further explored by evaluating the phonon DOS of H-graphene, DNTT and hydrogen with $10 \%, 50 \%$ and $100 \%$ hydrogen coverage ratio as shown in Fig. 5. It can be observed that by comparing phonon DOS of pristine graphene (Fig. 3) and H-graphene, the shape of in-plane, out-of-plane and total phonon DOS of graphene shows a remarkable deformation. Due to the light atom mass of hydrogen, the hydrogen atom vibrates at a high frequency as shown in Fig. 5(g)-(i). The hydrogen additionally broadens the total phonon DOS of graphene and brings about a high-frequency peak at approximately $87 \mathrm{THz}$ (Fig.5 (a)-(c)). Meanwhile, the G-band peak (around $47.7 \mathrm{THz}$ ) of graphene DOS is weakened and shows a shift to the high frequency for hydrogen coverage ratio $10 \%$ and 50\%. Based on Fig. 3(a) and Fig. 5(a)-(c), we can calculate the phonon overlapping factor $(\delta)$ of graphene and DNTT as $0.598,0.647,0.653$ and 0.580 for hydrogen coverage ratio $0 \%, 10 \%$ and $50 \%$ and $100 \%$, respectively. The phonon overlapping factor gives a direct explanation for TBR variation trend, which firstly drops, then saturates and finally increases. In supported graphene, due to the phonon scattering at supporting atoms, in-plane and out-of-plane phonons of graphene are coupled, and this coupling effect plays an important role in energy propagation. ${ }^{39,69}$ Zhang et al. has found that the energy conversion between in-plane transverse (TA) and longitudinal (LA) phonons was much faster than that between in-plane (TA/LA) and out-of-plane (ZA) phonons. ${ }^{5}$ Therefore, thermal resistance of the energy conversion in supported graphene majorly locates in the conversion between in-plane and out-ofplane phonons. Fig. 3(b) and Fig. 5(d)-(f) exhibit in-plane and out-of-plane phonon DOS of graphene. The adding of hydrogen atoms suppresses the G-band frequency of in-plane phonon mode, broadens the out-of-plane phonon mode, and shifts out-of-plane phonon mode to high frequency. The calculated $\delta$ of in-plane and out-of-plane phonons in graphene are $0.443,0.579,0.674$ and 0.713 for hydrogen coverage ratio $0 \%, 10 \%$, $50 \%$ and $100 \%$, respectively. These results indicate the hydrogen atoms generate more graphene phonon scattering centers, which will strengthen the energy conversion between in-plane and out-of-plane phonons in graphene. The improved energy 
conversion between in-plane and out-of-plane phonons is beneficial to heat transfer from in-plane to out-of-plane phonons in graphene, which indirectly enhances the interfacial thermal conductance between graphene and DNTT phonons. Moreover, the hydrogen atoms act as another participant to interact with atoms in DNTT molecules, which opens a new channel to transport energy across the interface. On the slight increase of TBR with hydrogen coverage ratio $80 \%-100 \%$, it is attributed to the reduction of phonon overlapping of graphene and DNTT. Additionally, as shown in Fig. 5(g)-(i), higher hydrogen coverage would make the DOS of hydrogen shift to high frequency, which also inhibits the direct energy transport from hydrogen to DNTT to some extent.

Similar to the effect of hydrogenation on the interfacial thermal conductance of a silicene/graphene bilayer investigated by Liu et al., ${ }^{39}$ this effect of hydrogenation on the TBR is further confirmed by alternately turning off the interaction of "C" or " $\mathrm{H}$ " with the atoms of DNTT. (See the Fig. S3 in the ESI†) We find out that when the interaction of "C" with DNTT is turned off, the TBR value is one order of magnitude larger. Besides, the contribution from " $\mathrm{C}$ " of hydrogenated graphene to interfacial thermal transport surpasses the direct effect from " $\mathrm{H}$ " of hydrogenated graphene. It is important to mention that although graphene hydrogenation reduces the thermal conductivity of graphene, ${ }^{34,35,64}$ it can still enhance interfacial thermal transport of graphene and DNTT. This enhancement can be summarized into three major reasons: (a) higher phonon transmission capacity between graphene and DNTT; (b) improved energy conversion between in-plane and out-of-plane phonons in graphene; (c) direct interaction of hydrogen atoms with atoms in DNTT molecules.

\subsection{Effect of vacancy defects}

During the vapor phase deposition of the graphene or organic semiconductors, vacancy defects due to the surface energy variation, impurities or roughness of the substrate are usually unavoidable. As these vacancies in the organic active layer will induce unpleasant effects on the device performance, ${ }^{70-72}$ it is worth to investigate their influence on the interfacial thermal transport. Firstly, we randomly delete some of the carbon atoms in graphene to create the single-vacancy defects in graphene while 
maintain the DNTT perfect. Then the DNTT molecules in the DNTT monolayer close to the interface are also randomly removed to create DNTT vacancy and graphene is kept pristine.

Fig. 6 exhibits the TBR variation with respect to the different graphene and DNTT vacancies. It can be noted that when graphene vacancy increases from $0 \%$ to $6 \%$, TBR will reduce from $5.07 \pm 0.35 \times 10^{-8} \mathrm{~m}^{2}-\mathrm{K} / \mathrm{W}$ to $3.06 \pm 0.08 \times 10^{-8} \mathrm{~m}^{2}-\mathrm{K} / \mathrm{W}$. This interesting observation suggests although graphene vacancies would induce the phonon scattering

in graphene sheet to block in-plane thermal transport of graphene, ${ }^{29,} 30$ the vacancies improve the out-of-plane interfacial thermal propagation. As shown in Fig. 7, similar to phonon DOS of hydrogenated graphene, graphene vacancies would broaden the phonon DOS of graphene and shift the G-band peak of graphene DOS, which enhances the overlapping areas of graphene and DNTT. In addition, graphene vacancy also increases the overlapping areas of in-plane and out-of-plane DOS, which also benefits phonon transmission capacity across the interface between graphene and DNTT. In contrast, the TBR does not show an obvious dependence on DNTT vacancy as shown in Fig. 6. TBR maintains a constant level when DNTT vacancy concentration changes from $0 \%$ to $6.25 \%$. We also calculate phonon DOS of total graphene, DNTT, in-plane graphene and out-of-plane graphene as shown in the Fig. S4 in the ESI† and they show similar forms to those of pristine graphene-DNTT structure without DNTT vacancy as shown Fig. 3. Therefore, although the thermal conductivity of organic semiconductors is susceptible to vacancies, ${ }^{45,51}$ DNTT vacancies cannot affect the interfacial thermal transport between graphene and DNTT.

\subsection{Effects of temperature and coupling strength}

In the real application of graphene-based electronic devices, due to the complicated thermal environment, the devices would operate at a temperature range. Hence temperature dependence of interfacial thermal transport of graphene and semiconductors are necessary to be studied to help to understand the thermal dissipating capability of devices. Additionally, the surface treatment in organic device fabrication is always used to modify the material interaction coupling at the heterojunction, ${ }^{71,73-75}$ which immensely affect the electrical performance of devices. In this section, we also 
investigate the effect of coupling strength of graphene and DNTT by controlling the coupling strength factor, $\chi$.

Firstly, we study the temperature dependence of TBR by tuning the equilibrium temperature in NVT ensemble ranging from $100 \mathrm{~K}$ to $600 \mathrm{~K}$. The coupling strength factor is still set to 1. As shown in Fig. 8, TBR would descend with the temperature increases. When the temperature is $600 \mathrm{~K}$, the TBR values of graphene-DNTT is $3.49 \pm 0.37 \times 10^{-8} \mathrm{~m}^{2}-\mathrm{K} / \mathrm{W}$, which is the $41.1 \%$ of TBR value at $100 \mathrm{~K}\left(8.50 \pm 0.20 \times 10^{-8}\right.$ $\left.\mathrm{m}^{2}-\mathrm{K} / \mathrm{W}\right)$. We attribute the reduction of TBR to the more phonon participation into interfacial thermal transport and stronger Umklapp phonon scattering. When temperature increases, more high-frequency phonon would be excited, ${ }^{39,}{ }^{49}$ which enlarges the phonon transmission channel across the graphene and DNTT. Furthermore, many theoretical and experimental work have demonstrated that high temperature results in the enhancement of the Umklapp phonon scattering, ${ }^{76}$ which plays a dominant role in interfacial thermal transport. ${ }^{77,78}$ Strong Umklapp scattering would shorten the phonon mean free path and decompose the high-frequency phonons into more lowfrequency phonons, which benefits the phonon coupling of graphene and DNTT as well as in-plane and out-of-plane phonon conversion of graphene. As a result, the temperature increase could make a significant contribution to the interfacial phonon propagation across graphene and DNTT.

The coupling strength is another key factor to affect the TBR of graphene and DNTT. In the simulation, we change the coupling strength factor of van der Waals interaction of graphene and DNTT varying from 0.5 to 3.0. From Fig. 9, the TBR value of graphene and DNTT shows a monotonous downtrend. When the coupling strength increases from 0.5 to 3 , the TBRs can drop around $86.5 \%$. Because the interaction between graphene and organic semiconductor mainly depends on the van der Waals forces of materials, the enhancement of the coupling strength factor will reinforce the interaction of graphene and DNTT, which further improves the energy transport capability across the interface. It is demonstrated that the surface treatment during the device fabrication not only affects the electrical properties, but also exerts the impact on the interfacial thermal transport. 


\section{Conclusions}

The energy transport at the interfaces between the graphene and organic semiconductors are playing a critical role in performance and lifetime of the graphenebased organic electronic devices. In this study, we apply molecular dynamics simulation to study interfacial thermal transport across graphene and organic semiconductor, dinaphtho[2,3-b:2',3'-f]thieno[3,2-b]thiophene (DNTT). The average TBR of graphene and DNTT is $4.88 \pm 0.12 \times 10^{-8} \mathrm{~m}^{2}-\mathrm{K} / \mathrm{W}$, which does not show the obvious dependence on graphene and DNTT size. We find that the hydrogenation of graphene is able to facilitate the heat transport across graphene and DNTT and TBRs can drop to around $8.4 \times 10^{-9} \mathrm{~m}^{2}-\mathrm{K} / \mathrm{W}$ after graphene hydrogenation. which is attributed to enhanced phonon coupling of graphene and DNTT, better energy conversion of graphene in-plane and out-of-plane phonons and direct interaction of hydrogen with DNTT. After adding the vacancy defects into DNTT and graphene, DNTT vacancy defects do not exert significant effect on TBR while graphene vacancy defects can reduce from $5.07 \pm 0.35 \times 10^{-8} \mathrm{~m}^{2}-\mathrm{K} / \mathrm{W}$ to $3.06 \pm 0.08 \times 10^{-8} \mathrm{~m}^{2}-\mathrm{K} / \mathrm{W}$ as graphene vacancy increases from $0 \%$ to $6 \%$. Furthermore, we find both temperature and coupling strength can reduce the TBR and benefit the interfacial thermal transport of graphene and DNTT. Our investigation about the interfacial thermal transport between graphene and organic semiconductors provides the fundamental knowledge to design and development of graphene-based organic semiconductors.

\section{Acknowledgements}

We greatly appreciate the support from General Research Fund (GRF) under Grant No. HKU 710313E and 17200314, Collaborative Research Fund (CRF) under Grant No. C704514E, the National Natural Science Foundation of China (NSFC) and the Research Grants Council (RGC) of Hong Kong Joint Research Scheme under Grant No. HKU 715/14. This research is partially conducted by using the research computing facilities and advisory services offered by Information Technology Services at The University of Hong Kong.

\section{References}


1. Y. Zhang, Y.-W. Tan, H. L. Stormer and P. Kim, Nature, 2005, 438, 201-204.

2. K. S. Novoselov, A. K. Geim, S. V. Morozov, D. Jiang, Y. Zhang, S. V. Dubonos, I. V. Grigorieva and A. A. Firsov, Science, 2004, 306, 666-669.

3. J. Zhang, F. Xu, Y. Hong, Q. Xiong and J. Pan, RSC Adv., 2015, 5, 89415-89426.

4. L. Lindsay, D. A. Broido and N. Mingo, Phys. Rev. B, 2010, 82, 115427.

5. J. Zhang, X. Wang and H. Xie, Phys. Lett. A, 2013, 377, 721-726.

6. A. A. Balandin, S. Ghosh, W. Bao, I. Calizo, D. Teweldebrhan, F. Miao and C. N. Lau, Nano Lett., 2008, 8, 902-907.

7. Y.-M. Lin, K. A. Jenkins, A. Valdes-Garcia, J. P. Small, D. B. Farmer and P. Avouris, Nano Lett., 2009, 9, 422-426.

8. Y.-M. Lin, C. Dimitrakopoulos, K. A. Jenkins, D. B. Farmer, H.-Y. Chiu, A. Grill and P. Avouris, Science, 2010, 327, 662.

9. X. Xu, L. F. C. Pereira, Y. Wang, J. Wu, K. Zhang, X. Zhao, S. Bae, C. Tinh Bui, R. Xie, J. T. L. Thong, B. H. Hong, K. P. Loh, D. Donadio, B. Li and B. Özyilmaz, Nat. Commun., 2014, 5, 3689.

10. B. D. Kong, S. Paul, M. B. Nardelli and K. W. Kim, Phys. Rev. B, 2009, 80, 033406.

11. J. Cai, P. Ruffieux, R. Jaafar, M. Bieri, T. Braun, S. Blankenburg, M. Muoth, A. P. Seitsonen, M. Saleh, X. Feng, K. Mullen and R. Fasel, Nature, 2010, 466, 470-473.

12. T. Ohta, A. Bostwick, T. Seyller, K. Horn and E. Rotenberg, Science, 2006, 313, 951-954.

13. G. Fiori, S. Lebègue, A. Betti, P. Michetti, M. Klintenberg, O. Eriksson and G. Iannaccone, Phys. Rev. B, 2010, 82, 153404.

14. A. K. Singh and B. I. Yakobson, Nano Lett., 2009, 9, 1540-1543.

15. J. Park, W. H. Lee, S. Huh, S. H. Sim, S. B. Kim, K. Cho, B. H. Hong and K. S. Kim, J. Phys. Chem. Lett., 2011, 2, 841-845.

16. Y. Wang, X. Chen, Y. Zhong, F. Zhu and K. P. Loh, Appl. Phys. Lett., 2009, 95, 063302.

17. W. Hong, Y. Xu, G. Lu, C. Li and G. Shi, Electrochem. Commun., 2008, 10, 
$1555-1558$.

18. J. Huang, D. R. Hines, B. J. Jung, M. S. Bronsgeest, A. Tunnell, V. Ballarotto, H. E. Katz, M. S. Fuhrer, E. D. Williams and J. Cumings, Org. Electron., 2011, 12, 1471-1476.

19. S. Chung, J.-H. Lee, J. Jeong, J.-J. Kim and Y. Hong, Appl. Phys. Lett., 2009, 94, 253302 .

20. C. Pannemann, T. Diekmann and U. Hilleringmann, J. Mater. Res., 2004, 19, 1999-2002.

21. E. Vitoratos, S. Sakkopoulos, E. Dalas, N. Paliatsas, D. Karageorgopoulos, F. Petraki, S. Kennou and S. A. Choulis, Org. Electron., 2009, 10, 61-66.

22. J. Zhang, Y. Wang and X. Wang, Nanoscale, 2013, 5, 11598-11603.

23. Y. Hong, J. Zhang and X. C. Zeng, Phys. Chem. Chem. Phys., 2016, 18, 2416424170.

24. M. Li, J. Zhang, X. Hu and Y. Yue, Appl. Phys. A, 2015, 119, 415-424.

25. Y. Hong, L. Li, X. C. Zeng and J. Zhang, Nanoscale, 2015, 7, 6286-6294.

26. Z. Chen, W. Jang, W. Bao, C. N. Lau and C. Dames, Appl. Phys. Lett., 2009, 95, 161910 .

27. Y. Wang, A. K. Vallabhaneni, B. Qiu and X. Ruan, Nanosc. Microsc. Therm., 2014, 18, 155-182.

28. Y. Wang, S. Chen and X. Ruan, Appl. Phys. Lett., 2012, 100, 163101.

29. H. Zhang, G. Lee and K. Cho, Phys. Rev. B, 2011, 84, 115460.

30. F. Hao, D. Fang and Z. Xu, Appl. Phys. Lett., 2011, 99, 041901.

31. J.-W. Jiang, J. Lan, J.-S. Wang and B. Li, J. Appl. Phys., 2010, 107, 054314.

32. J. Hu, S. Schiffli, A. Vallabhaneni, X. Ruan and Y. P. Chen, Appl. Phys. Lett., 2010, 97, 133107.

33. Y. Tang, J. Li, X. Wu, Q. Liu, Y. Liu and P. Yang, Appl. Surf. Sci., 2016, 362, 86-92.

34. Q.-X. Pei, Z.-D. Sha and Y.-W. Zhang, Carbon, 2011, 49, 4752-4759.

35. S.-K. Chien, Y.-T. Yang and C. o.-K. Chen, Appl. Phys. Lett., 2011, 98, 033107.

36. S.-K. Chien, Y.-T. Yang and C. o.-K. Chen, Carbon, 2012, 50, 421-428. 
37. S. Lin and M. J. Buehler, Nanotechnology, 2013, 24, 165702.

38. B. Liu, J. A. Baimova, C. D. Reddy, S. V. Dmitriev, W. K. Law, X. Q. Feng and K. Zhou, Carbon, 2014, 79, 236-244.

39. B. Liu, J. A. Baimova, C. D. Reddy, A. W.-K. Law, S. V. Dmitriev, H. Wu and K. Zhou, ACS Appl. Mater. Interfaces, 2014, 6, 18180-18188.

40. Y. Hong, J. Zhang and X. C. Zeng, Nanoscale, 2016, 8, 19211-19218.

41. Y. Hong, C. Zhu, M. Ju, J. Zhang and X. C. Zeng, Phys. Chem. Chem. Phys., 2017, 19, 6554-6562.

42. B. Liu, F. Meng, C. D. Reddy, J. A. Baimova, N. Srikanth, S. V. Dmitriev and K. Zhou, RSC Adv., 2015, 5, 29193-29200.

43. W. Xie, K. Willa, Y. Wu, R. Häusermann, K. Takimiya, B. Batlogg and C. D. Frisbie, Adv. Mater., 2013, 25, 3478-3484.

44. X. Wang, K. D. Parrish, J. A. Malen and P. K. L. Chan, Sci. Rep., 2015, 5, 16095.

45. X. Wang, J. Zhang, Y. Chen and P. K. L. Chan, Nanoscale, 2017, 9, 2262-2271.

46. S. Plimpton, J. Comput. Phys., 1995, 117, 1-19.

47. W. B. Donald, A. S. Olga, A. H. Judith, J. S. Steven, N. Boris and B. S. Susan, J. Phys. Condens. Matter, 2002, 14, 783.

48. J. Zhang and X. Wang, Nanoscale, 2013, 5, 734-743.

49. J. Zhang, Y. Hong and Y. Yue, J. Appl. Phys., 2015, 117, 134307.

50. J. Wang, R. M. Wolf, J. W. Caldwell, P. A. Kollman and D. A. Case, J. Comput. Chem., 2004, 25, 1157-1174.

51. D. Wang, L. Tang, M. Long and Z. Shuai, J. Phys. Chem. C, 2011, 115, 59405946.

52. M. Yoneya, M. Kawasaki and M. Ando, J. Mater. Chem., 2010, 20, $10397-$ 10402.

53. W. Shi, J. Chen, J. Xi, D. Wang and Z. Shuai, Chem. Mater., 2014, 26, 26692677.

54. A. K. Rappe, C. J. Casewit, K. S. Colwell, W. A. Goddard and W. M. Skiff, J. Am. Chem. Soc., 1992, 114, 10024-10035.

55. T. Yamamoto and K. Takimiya, J. Am. Chem. Soc., 2007, 129, 2224-2225. 
56. J. Zhang, Y. Hong, Z. Tong, Z. Xiao, H. Bao and Y. Yue, Phys. Chem. Chem. Phys., 2015, 17, 23704-23710.

57. J. Zhang, Y. Hong, M. Liu, Y. Yue, Q. Xiong and G. Lorenzini, Int. J. Heat Mass Transfer, 2017, 104, 871-877.

58. H. Zhong and J. R. Lukes, Phys. Rev. B, 2006, 74, 125403.

59. D. L. Nika, A. S. Askerov and A. A. Balandin, Nano Lett., 2012, 12, 3238-3244.

60. T. Luo and J. R. Lloyd, Adv. Funct. Mater., 2012, 22, 2495-2502.

61. Y. Liu, C. Hu, J. Huang, B. G. Sumpter and R. Qiao, J. Chem. Phys., 2015, 142, 244703.

62. Y. Liu, J. Huang, B. Yang, B. G. Sumpter and R. Qiao, Carbon, 2014, 75, 169177.

63. Q. X. Pei, Y. W. Zhang and V. B. Shenoy, Carbon, 2010, 48, 898-904.

64. B. Liu, C. D. Reddy, J. Jiang, J. A. Baimova, S. V. Dmitriev, A. A. Nazarov and K. Zhou, Appl. Phys. Lett., 2012, 101, 211909.

65. J. Zhou, Q. Wang, Q. Sun, X. S. Chen, Y. Kawazoe and P. Jena, Nano Lett., 2009, 9, 3867-3870.

66. R. R. Nair, W. Ren, R. Jalil, I. Riaz, V. G. Kravets, L. Britnell, P. Blake, F. Schedin, A. S. Mayorov, S. Yuan, M. I. Katsnelson, H.-M. Cheng, W. Strupinski, L. G. Bulusheva, A. V. Okotrub, I. V. Grigorieva, A. N. Grigorenko, K. S. Novoselov and A. K. Geim, Small, 2010, 6, 2877-2884.

67. D. W. Boukhvalov, M. I. Katsnelson and A. I. Lichtenstein, Phys. Rev. B, 2008, 77, 035427.

68. D. C. Elias, R. R. Nair, T. M. G. Mohiuddin, S. V. Morozov, P. Blake, M. P. Halsall, A. C. Ferrari, D. W. Boukhvalov, M. I. Katsnelson, A. K. Geim and K. S. Novoselov, Science, 2009, 323, 610-613.

69. J. H. Seol, I. Jo, A. L. Moore, L. Lindsay, Z. H. Aitken, M. T. Pettes, X. Li, Z. Yao, R. Huang, D. Broido, N. Mingo, R. S. Ruoff and L. Shi, Science, 2010, 328, 213-216.

70. A. Poschlad, V. Meded, R. Maul and W. Wenzel, Nanoscale Res. Lett., 2012, 7, 1-5. 
71. R. Ruiz, D. Choudhary, B. Nickel, T. Toccoli, K.-C. Chang, A. C. Mayer, P. Clancy, J. M. Blakely, R. L. Headrick, S. Iannotta and G. G. Malliaras, Chem. Mater, 2004, 16, 4497-4508.

72. S. Seo, L. C. Grabow, M. Mavrikakis, R. J. Hamers, N. J. Thompson and P. G. Evans, Appl. Phys. Lett., 2008, 92, 153313.

73. B. Nickel, R. Barabash, R. Ruiz, N. Koch, A. Kahn, L. C. Feldman, R. F. Haglund and G. Scoles, Phys. Rev. B, 2004, 70, 125401.

74. H. Chang, Y. Deng, Y. Geng, T. Wang and D. Yan, Org. Electron., 2015, 22, 8691.

75. M.-C. Jung, M. R. Leyden, G. O. Nikiforov, M. V. Lee, H.-K. Lee, T. J. Shin, K. Takimiya and Y. Qi, ACS Appl. Mater. Interfaces, 2015, 7, 1833-1840.

76. M. G. Holland, Phys. Rev., 1963, 132, 2461-2471.

77. P. E. Hopkins, P. M. Norris and R. J. Stevens, J. Heat Transfer, 2008, 130, 022401 .

78. P. E. Hopkins and P. M. Norris, J. Heat Transfer, 2009, 131, 022402. 


\section{Figures}

(a)

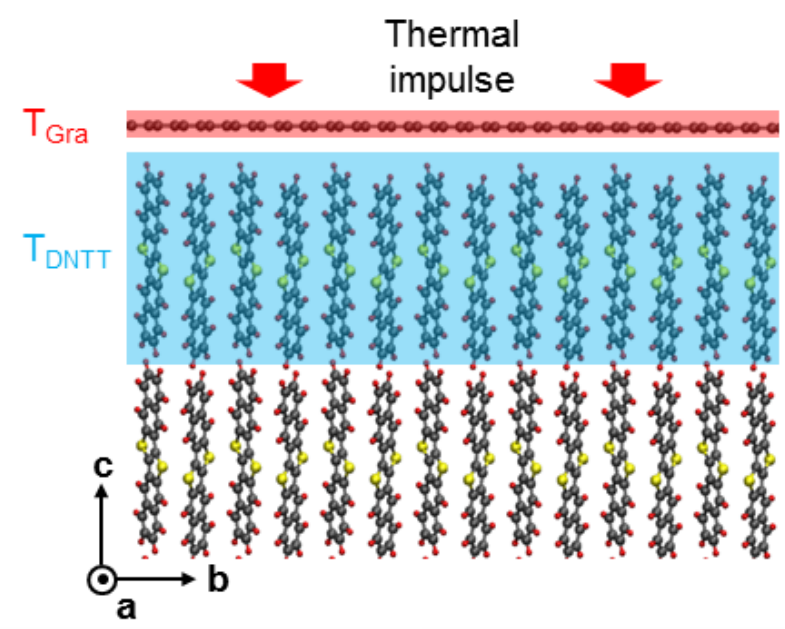

(b) $\widehat{\widehat{D}}-3.530 \square 600$

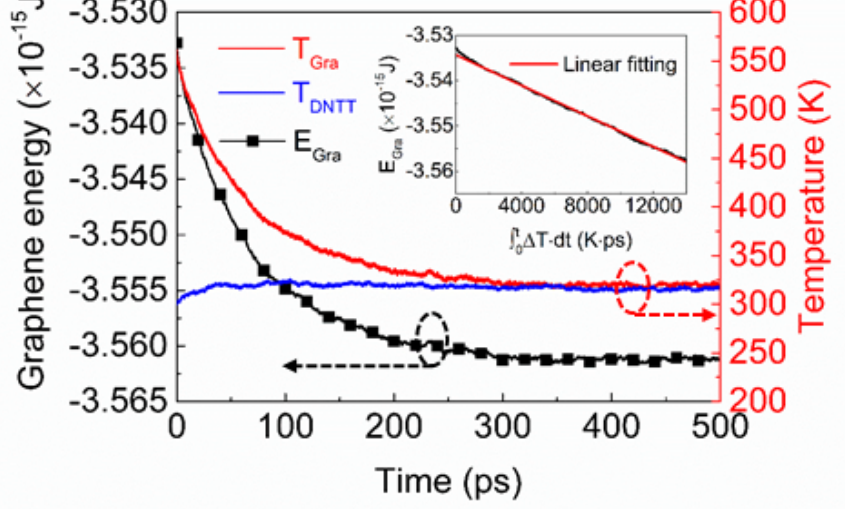

Fig. 1 (a) Schematic illustration of graphene-DNTT heterostructure. A 50 fs thermal impulse is inputted on graphene. The temperature of graphene (red region), $T_{G r a}$, and temperature of DNTT monolayer close to the interface (blue region), $T_{D N T T}$, are recorded as a function of time. (b) The energy evolution of graphene during $500 \mathrm{ps}$ relaxation process (left $y$-axis) and the temperature evolution of graphene and single DNTT layer during 500 ps relaxation process (right $y$-axis), The inset figure shows the linear fitting of energy with temperature difference integration based on Eq. (3). 


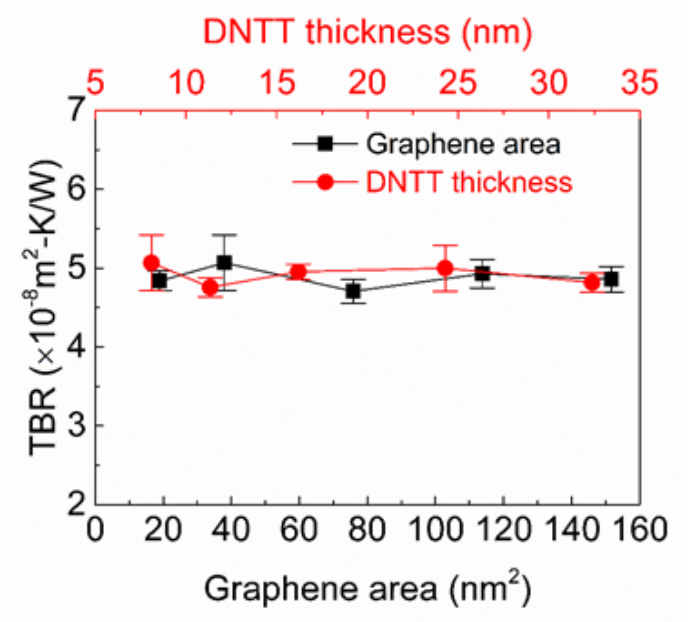

Fig. 2 TBR variation of graphene and DNTT at different graphene areas and DNTT thicknesses. 


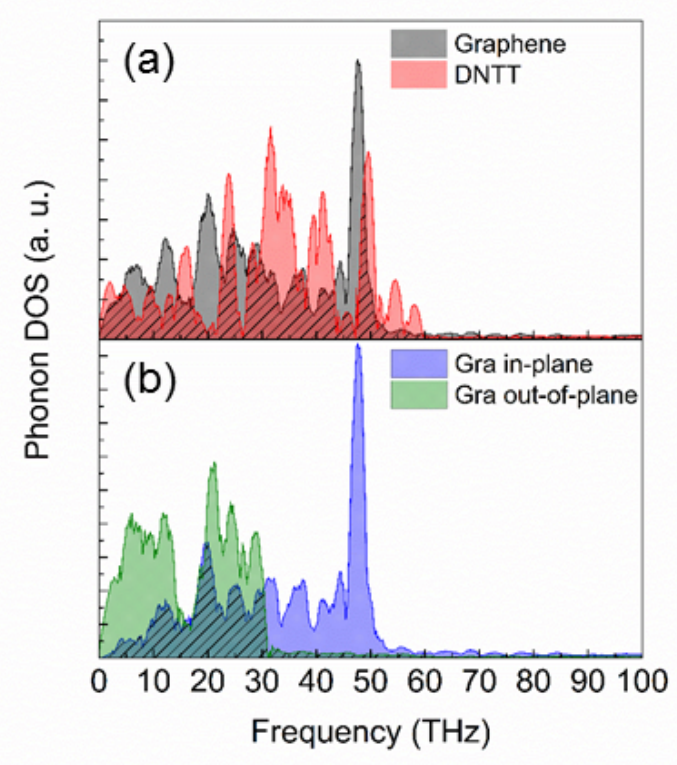

Fig. 3 (a) Phonon DOS of total graphene and DNTT. (b) Phonon DOS of in-plane and out-of-plane graphene. Slanted line areas denote overlap of phonon DOS. The phonon overlapping factor of graphene and DNTT is 0.598 ; and the phonon overlapping factor of in-plane and out-of-plane phonons in graphene is 0.443 . 


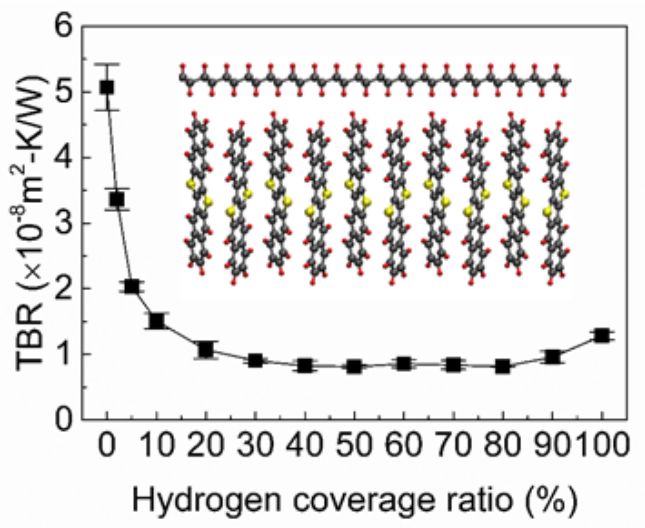

Fig. 4 TBR variation of graphene and DNTT at different hydrogen coverage ratios. The inset figure shows the illustration of hydrogenated graphene-DNTT heterostructure. 

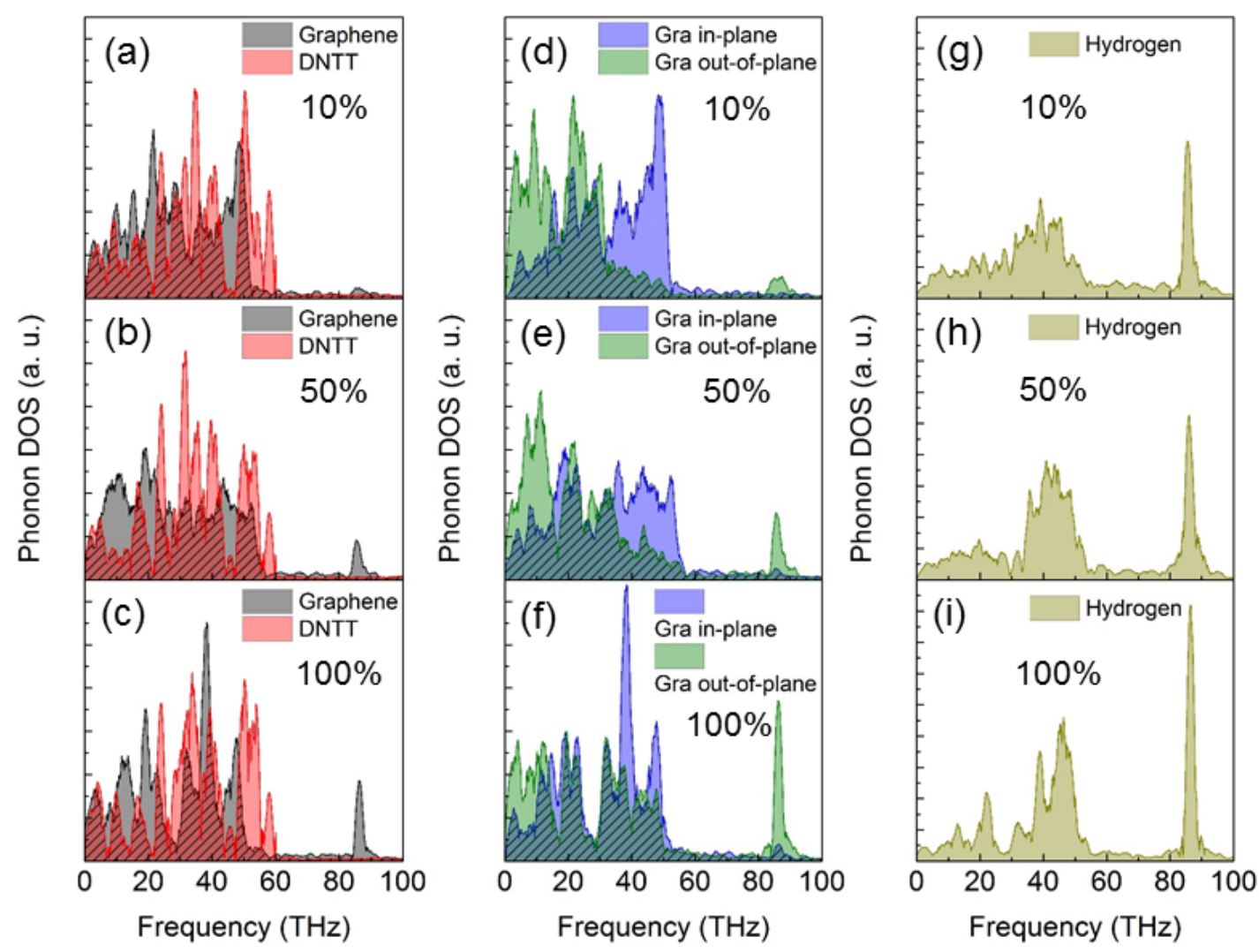

Fig. 5 (a)-(c) Phonon DOS of total graphene and DNTT at hydrogen coverage ratio of $10 \%, 50 \%$ and $100 \%$. (d)-(f) Phonon DOS of in-plane and out-of-plane graphene at hydrogen coverage ratio of $10 \%, 50 \%$ and $100 \%$. (g)-(i) Phonon DOS of hydrogen atoms at hydrogen coverage ratio of $10 \%, 50 \%$ and $100 \%$. Slanted line areas denote overlap of phonon DOS. The phonon overlapping factors of graphene and DNTT are $0.647,0.653$ and 0.580 at hydrogen coverage ratio of $10 \%, 50 \%$ and $100 \%$; and the phonon overlapping factors of in-plane and out-of-plane phonons in graphene are 0.579 , 0.674 and 0.713 at hydrogen coverage ratio of $10 \%, 50 \%$ and $100 \%$. 


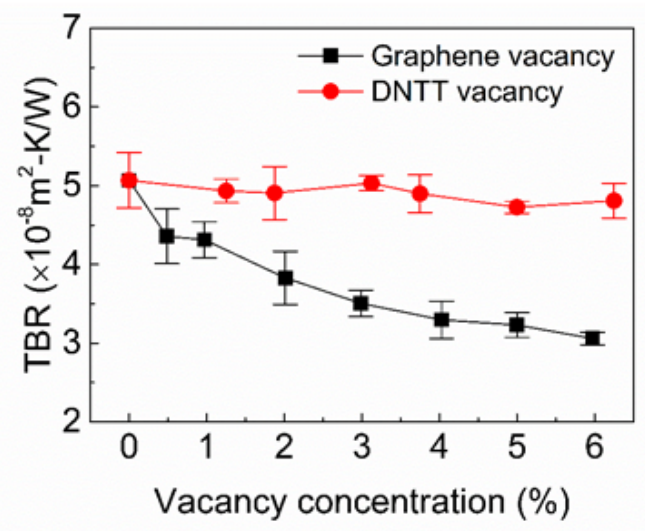

Fig. 6 TBR variation of graphene and DNTT at different graphene and DNTT vacancy concentrations. 

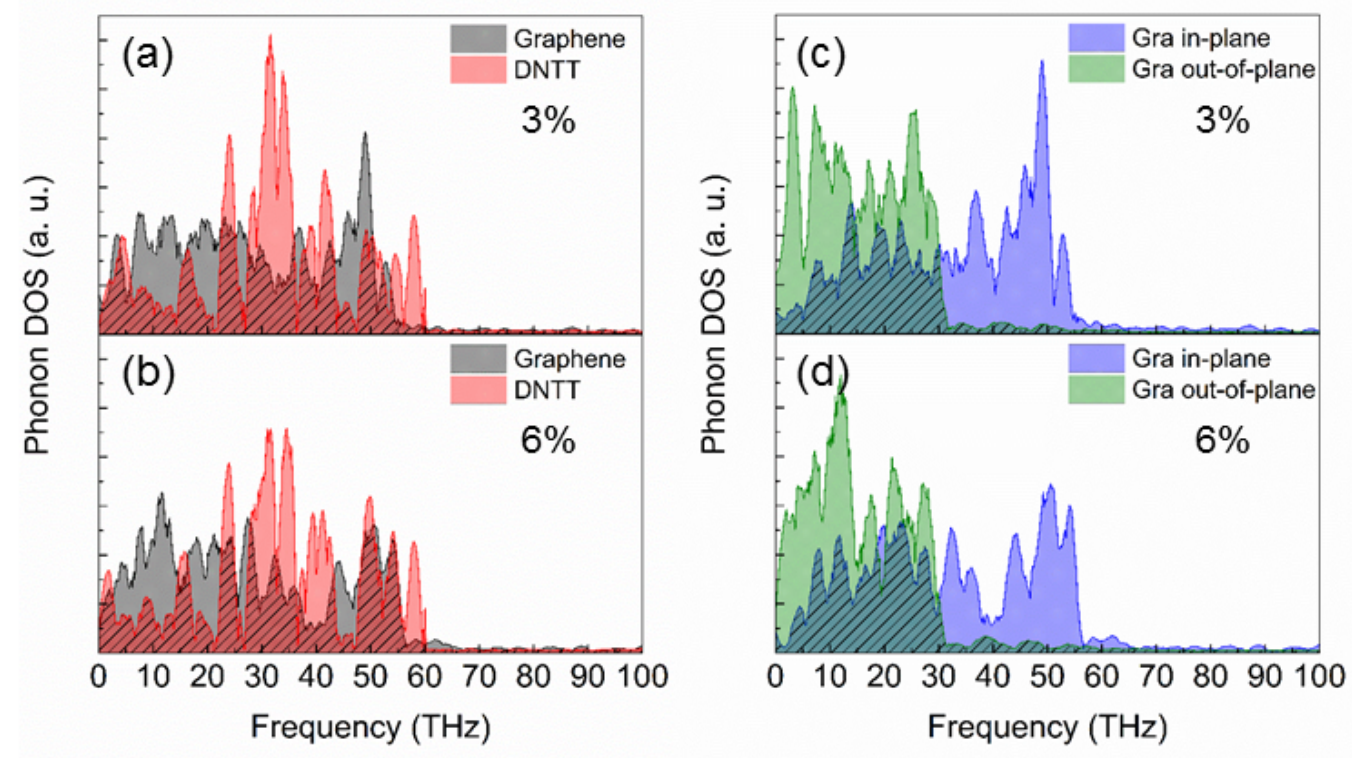

Fig. 7 (a)-(b) Phonon DOS of total graphene and DNTT at graphene vacancy concentration of 3\% and 6\%. (c)-(d) Phonon DOS of in-plane and out-of-plane graphene at graphene vacancy concentration of 3\% and 6\%. Slanted line areas denote overlap of phonon DOS. The phonon overlapping factors of graphene and DNTT are 0.633 and 0.643 at graphene vacancy concentration of $3 \%$ and $6 \%$; and the phonon overlapping factors of in-plane and out-of-plane phonons in graphene are 0.489 and 0.501 at graphene vacancy concentration of $3 \%$ and $6 \%$. 


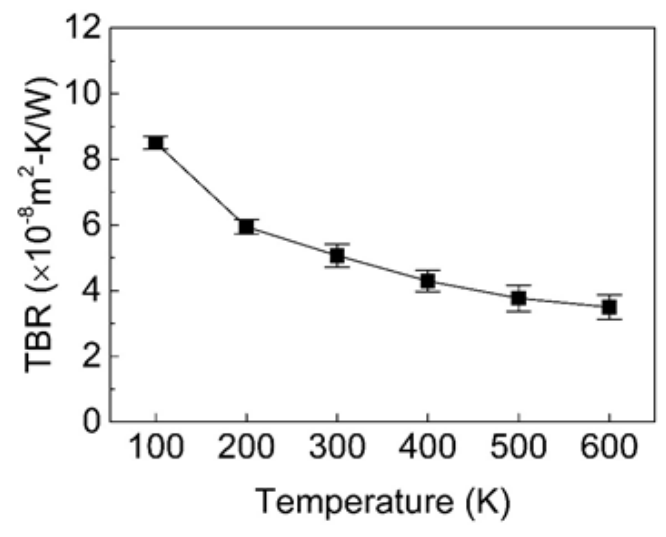

Fig. 8 TBR variation of graphene and DNTT at different temperatures from $100 \mathrm{~K}$ to $600 \mathrm{~K}$. 


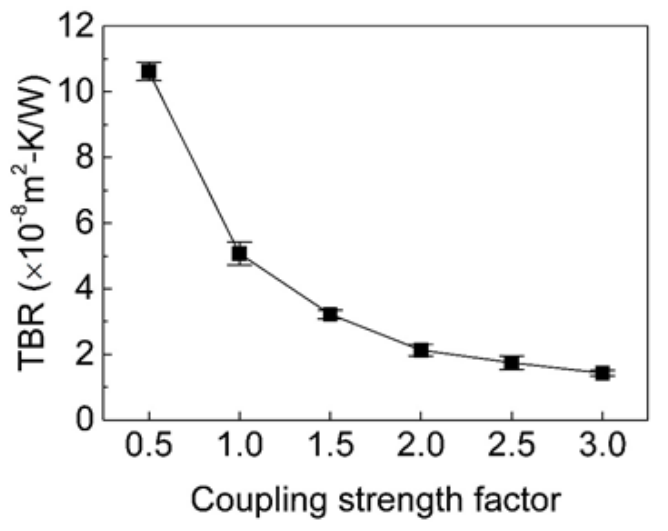

Fig. 9 TBR variation of graphene and DNTT at different coupling strength factors from 0.5 to 3.0 . 